\title{
Os rastros digitais na circulação de sentidos: pela desnaturalização e contextualização de dados na pesquisa em comunicação
}

\author{
Rafael Grohmann' \\ https://orcid.org/0000-0003-1063-8668 \\ I - Unisinos \\ São Leopoldo (RS). Brasil
}

Resumo: $\mathrm{O}$ artigo discute a circulação de sentidos como uma perspectiva teórico-metodológica para pesquisas em mídias sociais que busca a desnaturalização e a contextualização de dados. Isso envolve reincorporar os sujeitos com seus contextos, valores e contradições, envoltos em relações de poder e disputas por sentido, considerando, inclusive, os limites da circulação. Em termos metodológicos, trata-se de uma apreensão a partir dos rastros digitais, procurando ir além do descritivismo.

Palavras-chave: circulação; dados; metodologia; mídias sociais; sentidos.

Abstract: Digital Traces in the Circulation of Meanings: denaturalization and contextualization of data in communication research - The paper discusses the circulation of meanings as a theoreticalmethodological perspective for research in social media, in the sense of denaturalization and contextualization of data. This involves reincorporating subjects with their contexts, values and contradictions, involved in power relations and struggles for meaning, considering the limits of circulation. In methodological terms, it is an apprehension from the digital traces, trying to go beyond descriptivism.

Keywords: circulation; data; meanings; methodology; social media.

\section{Introdução}

Em 2008, Chris Anderson (2008) publicou na Wired o texto "O Fim da Teoria". Para ele, com a abundância de dados disponíveis aos acadêmicos, o trabalho científico 
seria tão somente o de interpretar o grande volume de dados disponível. Atualmente, com a relativa facilidade de "coleta de dados" em mídias sociais, há quem reproduza a ideia de que basta ter os dados - por exemplo, sobre alguma hashtag no Twitter - para produzir uma pesquisa. Van Dick (2014) considera que a dataficação é normalizada e naturalizada como um novo paradigma científico, transformando-se em uma ideologia.

Essa dataficação, colocada por Couldry e Hepp (2016) como midiatização profunda, pode ser definida como a crescente centralidade dos dados na vida cotidiana, afetando os processos comunicacionais, e também a pesquisa acadêmica sobre esses processos. Há um mantra que vem sendo repetido no campo dos negócios: "os dados são o novo petróleo". Couldry e Mejias (2018) justificam que os dados não são o novo petróleo a partir do conceito de colonialismo de dados, pois os dados não são produtos naturais, mas precisam ser produzidos e apropriados, no que Morozov (2018) chama de extrativismo de dados.

A partir deste contexto, o objetivo central deste artigo é problematizar qual pode ser um caminho possível, como uma proposta de agenda, para desnaturalizar e contextualizar, em perspectiva teórico-metodológica, dados de pesquisas em mídias sociais desde um ponto de vista crítico. Consideramos, pois, a perspectiva da circulação de sentidos para contextualizar e procurar dar sentido aos dados coletados. Com isso, a questão central é desdobrada nas seguintes propostas: a) a circulação de sentidos como lugar teóricoepistemológico desde o campo da comunicação, auxiliando a compreender processos comunicacionais nas mídias sociais; b) quais os desafios metodológicos para apreensão dos rastros digitais da circulação de sentidos em mídias sociais, procurando recontextualizar a produção de sentido dos sujeitos nessas ambiências (LIVINGSTONE, 2018).

\section{A circulação de sentidos na Comunicação}

A noção de circulação na comunicação pode abarcar distintos significados, desde visadas linguístico-discursivas (como discurso circulante em Charaudeau, 2006), passando por abordagens interacionais (BRAGA, 2006) até a circulação midiática, como o espalhamento midiático presente em Jenkins, Green e Ford (2014) ou a perspectiva de Fausto Neto (2010) acerca das zonas de contato/interpenetração em uma sociedade em vias de midiatização. A circulação também foi tematizada nos estudos culturais, a partir do circuito de cultura, como em Hall (2003), DuGay, Hall et al (1997) e Johnson (1999), como um olhar para compreender a totalidade do processo comunicacional, entre produção e recepção.

Dentre as diferentes abordagens para a noção de circulação, falamos aqui em circulação de sentidos, tal qual em Silverstone $(2002 ; 2006)$, como circulação de símbolos e significados por meio de processos comunicacionais, que são sociais e discursivos (por meio de interdiscursividades e intertextualidades, por exemplo). O foco nos sentidos nos diz que a circulação envolve mostrar como valores, visões de mundo e ideologias circulam 
nos processos comunicacionais. Desta forma, não nos referimos ao número de curtidas ou compartilhamento, ao nível de engajamento (GROHMANN, 2018) ou à chamada recirculação jornalística (ZAGO, 2015), ressaltando que a visada aqui levantada não é a única abordagem possível para a circulação na comunicação.

Na circulação, podemos observar como alguns sentidos são fixados ou ressignificados, revelando disputas e distinções no processo, como uma circulação de lutas por sentido (DYER-WITHEFORD, 1999; FAUSTO NETO, 2013). Como diz Fiske (1996, p. 143), "essa circulação social de sentidos sempre implica lutas e contestações, pois aqueles com poder social tentam constantemente reprimir, invalidar ou marginalizar significados produzidos por e que servem aos interesses de grupos subordinados". Isto é, não se trata aqui de analisar conversação em rede de análises interacionais, mas compreender como determinados significados são circulados em detrimento de outros, com distintos regimes de visibilidade.

Essa circulação de sentidos ocorre em diversos espaços: nas distintas plataformas, ruas e casas, com suas diferenças, semelhanças e desigualdades, em ambiências mediadas digitalmente ou não. Como afirma Couldry (2000, p. 78), "os sentidos circulam não somente em um meio, mas em vários, com inúmeras conexões entre eles". Contudo, conforme o autor, os sujeitos apenas acessam alguns sentidos circulantes. Nada (e ninguém) circula indefinidamente, pois há materialidades, limites e contradições na vida social e comunicativa. Isso significa dizer que pensar a circulação também envolve a "não circulação".

Essas disputas em torno da circulação nos mostram que há espacialidades e temporalidades envolvidas na circulação e suas interdições. Isso quer dizer que existem espaços e lugares de circulação, desde salões de cabelereiros até o WhatsApp, considerando a especificidade da circulação em cada lugar, tendo como exemplo as próprias plataformas digitais: a circulação que ocorre no Instagram é diferente da que acontece no YouTube. São circulações distintas marcadas também por lógicas algorítmicas. Uma hashtag circula de modos diferentes tanto nas distintas plataformas (Twitter e Instagram, por exemplo) quanto ao longo do tempo, desde horários e dias - com maior ou menor visualização e possibilidades de viralização - até sentidos distintos de uma hashtag de protesto, por exemplo, em anos diferentes, e o que elas significam no contexto macrossocial. São os contextos da circulação.

Podemos, então, considerar que há marcas da circulação a partir de dimensões espaciais, temporais e seus contextos sociais, inclusive ideológicos. Os contextos ajudam a desenhar lutas e embates em torno da circulação comunicacional, entre circulação e não circulação (impedimentos e interditos). São lutas por sentidos em circulação - produzidos, consumidos, ressemantizados e cristalizados.

O não acesso a esses sentidos pode ter diversas razões, tais como, mas sem a pretensão de esgotamento: a) marcadores estruturais como classe social, raça, gênero, sexualidade e geração, além dos posicionamentos ideológicos, considerando que somos marcados por essas identidades de maneira interseccional e não linear; b) de maneira específica, 
marcadores relacionados ao território, tais como país, estado, cidade e bairro; c) também de modo específico, marcadores dos distintos tipos de capital (o que, em alguma medida, pode também estar inserido na questão das classes sociais) - culturais, sociais, políticos, econômicos, midiáticos... - envolvendo composição, distribuição e volume (BOURDIEU, 2007); d) marcadores situacionais, não independentes dos outros fatores, mas que são complementares para entender os motivos de uma pessoa preferir ver uma série de drama ou comédia no Netflix, por exemplo; e) referente às práticas midiáticas, as possibilidades e limitações (ou as tais affordances) das plataformas digitais, em que a circulação de sentidos apresenta também uma dimensão algorítmica.

As práticas comunicacionais - seja um post no Facebook, uma conversa no bar ou o ato de ouvir uma música - nunca são ensimesmadas, pois se relacionam a outros processos envolvidos nas relações de poder da sociedade, que, por sua vez, estão inscritas nos processos comunicacionais. Quando consideramos as relações de poder nos processos comunicacionais, incluindo os signos ideológicos (VOLOCHINOV, 2017; FUCHS, 2017a), isso não significa reduzir a comunicação ao político ou ao sociológico. Trata-se de reinscrever os processos e contextos (macro) sociais na análise das atividades cotidianas de comunicação dos sujeitos.

Um dos desafios nesta perspectiva é o de posicionar o papel dos sujeitos e das instituições com relação à transformação de sentidos em sociedade. Por um lado, é preciso evitar naturalizar ou invisibilizar o papel da transformação nos processos comunicacionais, como se tudo fosse mera reprodução social. Por outro, é necessário evitar superestimar, romantizar ou idealizar possíveis "resistências" dos sujeitos. Pesquisas ligadas aos estudos culturais, como a de Hoggart (1973), por exemplo, já eram criticadas por certa romantização das resistências das classes trabalhadoras. Algo semelhante parece estar acontecendo na área de memes studies, superestimando o papel dos memes como protesto político (SHIFMAN, 2014; CHAGAS, 2019). Entre um caminho e outro, é preciso considerar as contradições nos processos, bem como as possibilidades, brechas e tentativas. Como mostra Livingstone (2018), a circulação de sentidos envolve processos que são, a um só tempo, "semioticamente abertos" e "estruturalmente desiguais".

Essa concepção de circulação de sentidos considera, pois, embates por significados e também por modos de circulação a partir de seus distintos contextos espaciais, temporais e sociais, reinserindo relações de poder e contextos (macro) sociais na análise dos processos comunicacionais.

Quando aplicada à análise de mídias sociais, a perspectiva da circulação de sentidos nem desconsidera a mercadorização e o trabalho gratuito dos usuários nas mídias sociais (FUCHS, 2017C), nem se reduz a essa perspectiva, no sentido de não resumir os sujeitos a dados (ou grafos, clusters...). Trata-se do reconhecimento das possibilidades de produção de sentido dos sujeitos nos distintos processos comunicacionais, considerando que o poder das plataformas digitais não é total, como mostra, por exemplo, a pesquisa de Bucher (2017) 
sobre a percepção de sujeitos "comuns" em relação aos algoritmos. Entre mercadorização/ exploração e as atividades cotidianas, os processos comunicacionais acontecem, com dilemas, contradições e valores. Isso significa reinserir questões ideológicas presentes nos processos comunicacionais na análise de mídias sociais.

Essa visão auxilia também na desnaturalização de termos como cultura da participação e cultura de fãs como se eles fossem automaticamente progressistas politicamente, como adverte Fuchs (2017c, p. 73) ao indagar: "o fascismo online é cultura participativa?". Para o autor, faltaria aos ideólogos da cultura da participação uma discussão sobre os potenciais pontos negativos de fãs e comunidades online, principalmente em um contexto político de ascensão da extrema-direita por todo o mundo. Da mesma forma, como mostram Dobson e Knezevic (2017), os memes podem ser tanto aspectos de ressignificação da cultura do remix quanto serem racistas e reproduzirem estereótipos de classe, raça e gênero.

Conforme Phillips e Milner (2017), a internet, assim como o mundo social e seus sujeitos, é feita de práticas ambivalentes: com criatividade e antagonismos, humor - nunca neutro - e injúrias. É palco, pois, de fãs de Star Wars, Anitta, Hitler (FUCHS, 2017) e também dos atiradores de Columbine (RICO, 2015). Também é cenário de criação de fanfictions de super-heróis do movimento Occupy (DE KOSNIK, 2017), em que super-heróis fictícios adentram o universo de movimentos sociais reais, transmutando "personagens populares em avatares de questões políticas específicas" (DE KOSNIK, 2017, P. 270).

Falar em circulação de sentidos nas mídias sociais, em uma perspectiva crítica, trata, pois, de compreender como são produzidos e circulados valores, ideologias, e como são as lutas por circulação, envolvidas em seus contextos sociais, espaciais e temporais também nas mídias sociais. Essa postura implica em não naturalizar noções ligadas à cultura digital, como atividades de fãs e memes, que, por vezes, são retratadas a partir de uma ótica somente celebratória. Compreender as contradições envolvidas na circulação de sentidos significa, por um lado, mostrar como há reiterações históricas nas manifestações em mídias sociais, mas também que ocorre "a expressão de novos e antigos sentidos, moldados por práticas novas e antigas, revelando novas possibilidades para a circulação dos sentidos na sociedade" (MATHIEU, 2015, p. 5)

Além dessa postura dialética, compreender a circulação de sentidos, com seus valores e embates, também passa por reinserir questões de classe, gênero e raça na análise de mídias sociais, como uma chave analítica para compreender: a) como as mensagens circuladas nas redes sociais digitais significam posições e relações de gênero, raça e classe; b) como a arquitetura das próprias plataformas digitais deixa entrever lógicas classistas, racistas e de gênero (NOBLE, 2018); c) a própria construção metodológica da pesquisa em mídias sociais, considerando que o Twitter, por exemplo, não pode ser tomado como reflexo de todo um país (SCHRADIE, 2017), isto é, como o próprio pesquisador, por vezes, naturaliza relações de raça, gênero e classe na produção e análise de pesquisa, faltando, pois, uma postura de vigilância e reflexividade epistemológica. 
A análise da circulação de sentidos em mídias sociais apresenta também, pois, uma questão metodológica: como apreender metodologicamente a circulação, considerando seus contextos e vestígios, e também os valores e embates em circulação?

\section{Desafios metodológicos: contextualização dos rastros digitais}

Os desafios de apreensão da circulação de sentidos em mídias sociais se relacionam a como desenhar metodologicamente a circulação em uma pesquisa empírica, considerando que não há como acessar sua totalidade e há limitações dos contextos de circulação, que já enunciamos acima, desde marcadores estruturais como classe, raça e gênero, passando por marcadores relacionados a território e distintos tipos de capitais até os situacionais. Envolve também, como afirmamos, as possibilidades e limitações das próprias plataformas digitais (SRNICEK, 2017), com suas mediações algorítmicas.

Mais do que um roteiro ou manual, refletimos a partir de algumas pistas metodológicas. Pieniz, Silva e Matos (2017), a partir de análise de teses e dissertações no âmbito da recepção em mídias sociais, afirmam que há certo descritivismo nas investigações, faltando conexões teórico-metodológicas mais profundas. Isso pode acontecer, por um lado, devido à dominância de grafos e estatísticas em pesquisas, havendo "poucas orientações para a compreensão das mídias sociais como um ambiente simbólico" (MATHIEU, 2015, p. 15). Por outro lado, uma análise de cunho mais qualitativo pode sofrer tanto de "frouxidão metodológica" quanto de falta de conexões do corpus analisado com os contextos de circulação.

Um dos desafios para a análise de circulação de sentidos é, então, reinserir as dinâmicas e os contextos socioculturais das coletas de dados em mídias sociais, afinal os sujeitos "são necessariamente sociais, envolvidos na sociedade e na história de diferentes maneiras" (LIVINGSTONE, 2018, p. 10). Para a autora, trata-se de envolver os sujeitos nos contextos de suas vidas, não bastando porcentagens ou observações descontextualizadas. Como ir além do que os dados dizem sobre os sujeitos (ATHIQUE, 2018)?

Isso passa, primeiramente, pelo reconhecimento da impossibilidade de analisar, nos limites de um artigo, todo o corpus coletado. Mais do que o próprio material levantado, é preciso saber o que fazer com ele: para quais propósitos coletei 20 mil tweets? A que eles servem à pesquisa? É ainda, então, o problema de pesquisa que guia o desenho metodológico, não podendo fetichizar a grande quantidade de dados para mineração na internet. Como afirma Malini (2017, p. 130), "saber questionar continua sendo o elemento mais importante da pesquisa. Não é o fetiche do volume que dá qualidade à pesquisa. Realmente, o que dá qualidade é a boa questão de pesquisa".

Propomos, então, como possibilidade metodológica para a circulação de sentidos, a quebra da Big Data em small data, ou seja, em volumes menores de dados que servem à questão da pesquisa. Isto pode ser mais interessante do que trabalhar com enormes 
volumes de dados dos quais não se consegue extrair explicações ou análises além das automáticas. Segundo Athique (2018, p. 72), "a real utilidade da Big Data não está em seu tamanho absoluto, mas no fato de ser granular", podendo, portanto, ser produtiva a operacionalização da pesquisa em escalas menores.

Se, pois, nunca é possível apreender toda a circulação de sentidos presentes na sociedade, e o que captamos são sempre rastros da circulação (com seus contextos e limitações), quando pesquisamos em mídias sociais, o que há é a busca por rastros digitais (BREITER; HEPP, 2018) na circulação de sentidos. Desenhar e pesquisar a circulação nos rastros digitais significa contextualizar os dados e processos comunicacionais, reconhecendo a complexidade das dinâmicas presentes.

Trata-se, pois, de pensar a circulação de sentidos em mídias sociais a partir da busca de indícios como processo metodológico, como aponta Braga (2008), procurando distinguir indícios essenciais de acidentais e tensionando mutuamente objeto empírico e teoria (ao contrário do que propunha Anderson, 2008). De acordo com Braga (2008, p. 86), "a derivação de conhecimento mais amplo com base em pesquisas indiciárias não se baseia na premissa de tipicidade ou de representatividade do caso singular - mas sim na constatação da possibilidade de existência do fenômeno". Com isso, "é possível, então, pesquisar e teorizar sobre as condições sociais dessa possibilidade" (Idem). O rigor (não rigidez) metodológico proposto pelo autor para a análise de indícios na comunicação nos auxilia a compreender os rastros digitais presentes na circulação de sentidos.

É necessário, então, também um questionamento (ou uma reflexividade) de ordem epistemológica em relação à coleta de dados em mídias sociais - a dimensão epistemológica do monitoramento online, como reivindicam Lopes e Freire (2012, p. 2): "Entendidas as técnicas como instrumentos neutros, naturalizados, facilmente intercambiáveis, a reflexividade sobre elas é débil exatamente por envolverem operações técnicas, isto é, supostamente 'não valorativas'". Nem a internet nem a pesquisa em internet são neutras.

O fetiche em torno da coleta de dados em mídias sociais reproduz naturalizações no processo de pesquisa, como mostra Van Dijck (2014). O descritivismo presente nas pesquisas não é só uma fragilidade metodológica mas também expressão de, nos termos de Mosco (2014), um positivismo digital. Podemos exemplificá-lo a partir da proposição de Anderson (2008) em relação ao fim da teoria ou dos que postulam que a coleta de dados consegue preceder quaisquer fundamentações teóricas, de forma neutra e imparcial, como determinadas perspectivas de grounded theory. O problema destas visões, segundo Fuchs (2017c, p. 57) é que "elas não conectam resultados estatísticos e computacionais à análise mais ampla de significados humanos, interpretações, atitudes, valores morais, dilemas éticos, usos, contradições e implicações macrossociológicas das mídias sociais".

Não se trata, aqui, de desqualificar métodos como análise de redes, cartografia das controvérsias e seus grafos (RECUERO, BASTOS, ZAGO, 2015; MALINI, 2016), mas de demonstrar que essas não são as únicas perspectivas metodológicas possíveis em 
pesquisas envolvendo mídias sociais, principalmente as que privilegiam a circulação de sentidos. Esse aprisionamento metodológico redunda em dificuldades para abordagens que privilegiam a circulação de sentidos. Como salienta Depexe (2017, p. 7), "os métodos empregados se aproximam da exploração científica e as análises culminam, geralmente, em um mapeamento temático ou categorização semântica". Ou seja, se por um lado, as ferramentas/softwares de coletas de dados auxiliam na pesquisa, não pode haver uma naturalização dos seus usos e protocolos.

Assim, os desafios do desenho metodológico da pesquisa sobre circulação de sentidos em mídias sociais referem-se a um recorte tanto do problema de pesquisa quanto do objeto empírico, de maneira a tentar apreender os rastros digitais da circulação, procurando não só descrevê-los, mas contextualizá-los em relação aos sujeitos envolvidos e também a limites e possibilidades dados pelas próprias plataformas digitais. Assim, os dados não circulam livremente pelas mídias sociais, mas também são recheados de contextos de circulação.

Na circulação de dados e métricas, a visibilidade e o poder, segundo Beer (2016), são tanto o produto quanto parte da maneira como os dados circulam. Desta forma, os métodos de mensuração desses rastros digitais não são neutros: "Os métodos eles próprios circulam pelo mundo social, assim como se movem entre setores e são implantados por diferentes e complexas configurações" (BEER, 2016, p. 81). Por isso, há de se entender os métodos como uma parte do sistema de mensuração e circulação dos próprios dados (SAVAGE, 2013; BEER, 2016). Nem os dados, nem os métodos circulam livremente. Não são naturais ou transparentes, mas tem, incluídos neles, determinados olhares, como o que Beer (2018) chama de data gaze.

Segundo Savage (2013), que também critica o positivismo na análise de dados, há uma política dos métodos, pois os métodos das ciências sociais, de certa maneira, são parte da racionalidade política de conhecer uma população: "Eu me distancio da visão de que os métodos são mais significativos hoje que no passado, no que pode ser visto como uma resposta à crescente política de [...] métricas" (SAVAGE, 2013, p. 4). Os métodos não são necessariamente sobre generalizações. Então, em conformidade com Braga (2008), podemos afirmar que a análise de rastros digitais presentes na circulação de sentidos não é algo envolto em generalizações, mas trata-se de "fazer inferências abstratas (genéricas) sobre o mundo em que aquele caso pode ocorrer" (BRAGA, 2008, p. 56). Como afirmamos anteriormente, os sujeitos e dados nunca são ensimesmados, mas se relacionam a outros contextos de circulação.

A perspectiva indiciária nos auxilia, por um lado, a ultrapassar o descritivismo, e, por outro, a inserir a agenda proposta em relação à circulação de sentidos, envolvendo relações de poder e ideologias circulantes. Como pesquisadores de comunicação, temos não somente de mostrar o que está acontecendo no Facebook ou no Instagram, mas o que aquelas manifestações significam para os processos comunicacionais em determinada sociedade. Quais são os valores e ideologias presentes nessa circulação de sentidos? Quais as lutas por sentido e os regimes de visibilidade? 
Na mesma direção, concordamos com Fuchs (2017b; 2017c) de que os métodos tradicionais das ciências sociais, embora não sejam únicos, não perderam importância na pesquisa em mídias sociais. Segundo o autor, "nós não precisamos apenas entender o que as pessoas fazem na internet, mas também [...] quais são as implicações mais amplas e como as estruturas de poder enquadram e moldam as atividades online" (FUCHS, 2017b, p. 43). Para Fuchs (2017b; 2017c), métodos digitais críticos aplicariam não apenas métodos quantitativos a partir do uso de ferramentas e softwares, mas também usariam amostras menores para análise qualitativa. Assim, o foco

pode, por exemplo, estar em tópicos-chave ou em usuários mais seguidos, com mais likes ou retweetados. Em alguns casos, a análise das publicações de usuários individuais pode ser mais apropriada; em outros casos, o foco pode estar em um período de tempo específico, em algum tópico do discurso, um grupo particular, características específicas, e assim por diante (FUCHS, 2017b, p. 44).

Como afirmamos anteriormente, o desenho de pesquisa está à serviço do problema de pesquisa. Desta forma, esses métodos podem ser articulados em "triangulação metodológica" (FIGARO, 2014) junto a outros, como entrevistas, grupos focais e/ou métodos com inspiração etnográfica, digital ou presencialmente, por exemplo. Métodos de análise, como análise de conteúdo ou análise de discurso, também não podem ser desprezados, desde que ajudem na contextualização dos dados coletados, além de apreender como são os valores e embates e circulação.

Há também de se compreender, conforme alerta Beer (2016), as possibilidades e restrições das próprias plataformas, a partir de seus "circuitos específicos de interação" (SBARDELOTTO, 2017) e da própria "gramaticalização técnica" (GERLITZ, RIEDER, 2018), ou ainda: como os feixes de sentido atualmente passam também por controle algorítmico das plataformas digitais? Assim, as próprias plataformas ajudam a configurar determinados modos de circulação, também desenhando rastros digitais específicos.

Esses contextos de circulação passam pelas restrições das Application Programming Interfaces (API), que ajudam a configurar modos de coleta dos dados e seus limites, e pelas especificidades dos distintos dispositivos comunicacionais. Isso significa considerar a gramaticalidade interna à própria plataforma (SRNICEK, 2017), com seus modos de produzir e fazer circular sentidos, "demarcando horizontes de ações possíveis (e impossíveis)" (GERLITZ; RIEDER, 2018, p. 531) em determinada plataforma. Envolve também os limites em relação à busca por palavras/metadados nas aproximações com o objeto empírico e as desigualdades inscritas no próprio desenho da plataforma, como tem afirmado as pesquisas de Eubanks (2018) e Noble (2018).

O que se evidencia, portanto, é uma busca metodológica por algo que não fique somente na descrição das atividades dos sujeitos, de forma a melhor apreender teóricometodologicamente a circulação de sentidos principalmente nos ambientes digitais; 
e de não recair em abordagens que redundem em um positivismo digital, pensando, pois, nas implicações da circulação de dados e métricas (BEER, 2016) para pesquisas sobre produção de sentidos envolvendo sujeitos.

Segundo Athique (2018), não se trata de nenhuma novidade segundo os antigos parâmetros dos estudos culturais, mas, no contexto das pesquisas envolvidas em métodos digitais, "o que nos resta é uma numerologia descontextualizada de forma radical e que apenas se inicia com o consumo online da cultura popular" (ATHIQUE, 2018, p. 73). Investigar os rastros digitais presentes na circulação de sentidos significa recontextualizar os dados e sujeitos considerando sua produção de sentidos em determinados contextos e regimes de visibilidade, envolvendo contradições e embates.

\section{Considerações finais}

Propomos como eixo central do presente artigo um caminho teórico-metodológico para problematizar e desnaturalizar condições de produção de dados e pesquisas em mídias sociais. Esse caminho simboliza mais uma agenda de possibilidades do que um roteiro fechado. Apresentamos a circulação de sentidos como uma proposta possível (não única) para a pesquisa em comunicação, como perspectiva que busca compreender: a) dialeticamente os processos comunicacionais, entre mudanças e permanências; b) construção e circulação de valores e ideologias, reincorporando as relações de poder e o político em perspectiva comunicacional; c) conexões entre o micro das atividades dos sujeitos no cotidiano a contextos (macro) sociais, sem cair em uma relação direta que não considere as complexidades dos processos comunicacionais; d) disputas por sentido envoltos na circulação comunicacional; e) contextos de circulação, desde questões espaciais e temporais, até questões de gênero, raça e classe, que ajudam a mostrar os alcances e limites da circulação.

Enquanto possibilidade teórico-metodológica para pesquisa em mídias sociais, isso significa considerar também os contextos de circulação dos dados (BEER, 2016), desde os sujeitos que inscrevem enunciados nas plataformas digitais até as políticas e gramáticas das próprias plataformas, envolvendo também lógicas algorítmicas na circulação de sentidos nestas redes sociais digitais. As mensagens no WhatsApp, por exemplo, obedecem a circuitos e lugares de enunciação específicos, que ajudam a circular determinados sentidos em detrimento de outros a partir dos processos comunicacionais ali estabelecidos.

Isso leva a desafios e a dilemas metodológicos de apreensão da circulação de sentidos em pesquisas envolvendo mídias sociais: Como coletar dados? Como analisá-los? Em primeiro lugar, há que desnaturalizar a produção e circulação dos dados, entendendo-os como parte de processos de apropriação e expropriação de sentidos (COULDRY, MEJIAS, 2018; MOROZOV, 2018). Isso significa dizer que os dados não são produzidos no vazio, mas em determinados contextos, não só a partir das lógicas das plataformas digitais, mas, 
neste caso, a partir de determinados enquadramentos do objeto empírico por visadas epistemológicas e teóricas. Assim, não é possível um dado se sustentar por si, como preconizava Anderson (2008). Essa desnaturalização auxilia a não cair em um fetiche da Big Data ou em um positivismo digital que apenas descrevem os dados existentes, como se houvesse um deslumbramento com o grande volume de dados coletados.

Defendemos, pois, que: a) o desenho da pesquisa deve sempre estar a mando do problema de pesquisa; b) não sendo possível a apreensão total da circulação de sentidos - em nenhuma ocasião -, é preciso criar estratégias metodológicas, com a devida reflexividade, para, de alguma maneira, sondar a circulação. Reconhecer os limites da circulação e sua apreensão metodológica nos ajuda a compreender que o que vemos são os rastros digitais (BREITER, HEPP, 2018) presentes na circulação de sentidos. Assim, uma perspectiva indiciária coloca-se como uma possibilidade, de maneira a tanto contextualizar os dados e sua circulação quanto a extrapolar o próprio material coletado (não enquanto generalização, mas como conexões).

Assim, é possível trabalhar com unidades menores de dados, de forma a serem devidamente contextualizados. Não se pode naturalizar, por exemplo, a circulação de sentidos no Twitter como se, de maneira direta, eles simbolizassem as dinâmicas sociais e comunicacionais da sociedade brasileira, sem levar em conta limites e contextos de circulação, inclusive de classes sociais.

A partir disso, mostram-se produtivos métodos clássicos das ciências sociais e da comunicação, como análise de conteúdo e análise de discurso, além da combinação com outros métodos de coleta de dados (entrevistas ou grupos focais). Métodos que são mainstream na pesquisa em mídias sociais, como análise de redes sociais ou cartografia de controvérsias não são os únicos existentes, mas também podem auxiliar a compor uma estratégia multimetodológica. Não se trata, porém, de desqualificação dessas abordagens, mas de mostrar que o velho não se perde em meio a situações comunicacionais colocadas como novas, podendo ter validade metodológica.

Por fim, trata-se de "reinserir os seres humanos, com toda sua complexidade redundante, bagunçada, vulnerável" (HUWS, 2011, p. 52) na pesquisa em mídias sociais, de maneira a entrever os sujeitos por meios dos rastros digitais presentes na circulação de sentidos - não para transformá-los novamente em dados, mas para extrair deles a sua humanidade, com suas contradições.

Rafael Grohmann é Professor do Programa de PósGraduação em Ciências da Comunicação da Universidade do Vale do Rio dos Sinos (Unisinos) e doutor em Ciências da Comunicação pela Universidade de São Paulo (USP).

rafael-ng@uol.com.br 


\section{Referências}

ANDERSON, C. The End of Theory: the data deluge makes the scientific method obsolet. Wired Magazine, junho 2008. Disponível em: <https://www.wired.com/2008/06/pb-theory/>. Acesso em: 17 fev. 2019.

ATHIQUE, A. The Dynamics and Potentials of Big Data for Audience Research. Media, Culture \& Society,.v. 40, n. 1, 2018.

BEER, D. Metric Power. Londres: Palgrave, 2016.

The Data Gaze. Londres: Sage, 2018.

BOURDIEU, P. A Distinção. São Paulo: Edusp, 2007.

BRAGA, J. L. A Sociedade Enfrenta sua Mídia. São Paulo: Paulus, 2006.

. Comunicação, disciplina indiciária. Revista MATRIZes. n. 2, abril 2008.

BREITER, A.; HEPP, A. The Complexity of Datafication: putting digital traces in context. In: HEPP, A.; BREITER, A.; HASEBRINK, U. (org.). Communicative Figurations. Londres: Palgrave, 2018. p. 387-405.

BUCHER, T. The Algorithmic Imaginary: exploring the ordinary affects of Facebook algorithms. Information, Communication \& Society, v. 20, n. 1, 2017.

CHAGAS, V. Digerindo o indigesto: a escatologia política do Vomitaço. Galáxia. N. 40, jan-abr. 2019, p. 41-56.

CharaudeaU, P. Discurso das Mídias. São Paulo: Contexto, 2006.

COULDRY, N. Inside Culture. Londres: Sage, 2000.

COULDRY, N.; HEPP, A. The Mediated Construction of Reality. Londres: Polity, 2017.

COULDRY, N.; MEJIAS, U. Data Colonialism: rethinking Big Data's relation to the contemporary subject. Television \& New Media, 2018.

DE KOSNIK, A. Memory, Archive and History in Political Fan Fiction. In: GRAY, J. et alli (org.). Fandom. 2a Edição. Nova lorque: NYU Press, 2017.

DEPEXE, S. Circulação de Salve Jorge no Twitter. Anais da Compós. São Paulo: Cásper Líbero, 2017. Disponível em: <http://www.compos.org.br/data/arquivos_2017/trabalhos_arquivo_SN543VIX13JY 997F9DR6_26_5100_21_02_2017_13_52_14.pdf >. Acesso em: 20 fev. 2018.

DOBSON, K.; KNEZEVIC, I. Liking and Sharing' the stigmatization of poverty and social welfare. TripleC, v. 15, n. 2, 2017.

DU GAY, P.; HALL, S. et al. Doing Cultural Studies: the story of the Sony Walkman. Londres: The Open University/ Sage, 1997.

DYER-WITHEFORD, N. Cyber-Marx. Chicago: University of Illinois Press, 1999.

EUBANKS, V. Automating Inequality. New York: St Martin's, 2018.

FAUSTO NETO, A. As bordas da circulação. Alceu, n. 20, v. 10, jan/jun 2010.

. Como as linguagens afetam e são afetadas na circulação? In: BRAGA, J. L.; FERREIRA,

J.; FAUSTO NETO, A.; GOMES, P. (org.). 10 Perguntas para a Produção de Conhecimento em Comunicação. São Leopoldo/RS: Ed. Unisinos, 2013, p. 43-64.

FIGARO, R. A triangulação metodológica em pesquisas sobre a comunicação no mundo do trabalho. Fronteiras, v. 16, n. 2, 2014. 
FISKE, J. British Cultural Studies and Television. In: STOREY, J. (org.). What is Cultural Studies? Londres: Arnold, 1996.

FUCHS, C. Fascism 2.0: Twitter users' social media memories of Hitler on his $127^{\text {th }}$ birthday. Fascism, v. 6,2017 .

From Digital Positivism and Administrative Big Data Analytics Towards Critical Digital and Social Media Research! European Journal of Communication, v. 32, n. 1, p. 37-49, 2017b.

Social Media: a critical introduction. $2^{\mathrm{a}}$ edição. Londres: Routledge, 2017c.

GERLITZ, C.; RIEDER, B. Tweets are not created equal: investigating Twitter's client ecosystem. International Journal of Communication, v. 12, 2018.

GROHMANN, R. A noção de engajamento: sentidos e armadilhas para a pesquisa em comunicação. FAMECOS, v. 25, n. 3, 2018.

HALL, S. Codificação/ Decodificação. In: Da Diáspora. Belo Horizonte: Ed. UFMG, 2003, p. 365-384. HOGGART, R. As Utilizações da Cultura. Lisboa: Ed. Presença, 1973.

HUWS, U. Mundo material: o mito da economia imaterial. Mediações, v. 16, n. 1, jan/jun 2011, p. 24-54.

JENKINS, H.; GREEN, J. ; FORD, S. Cultura da Conexão. São Paulo: Aleph, 2014.

JOHNSON, R. O que é, afinal, estudos culturais? In: SILVA, T. (org.) O que é, afinal, estudos culturais? Belo Horizonte: Autêntica, 1999.

LIVINGSTONE, S. Audiences in a Age of Datafication: critical questions for media research. Television \& New Media, 2018

LOPES, M. I. V. ; FREIRE, C. A dimensão epistemológica do monitoramento on-line. Anais da Compós. Juiz de Fora, 2012.

MALINI, F. Um método perspectivista de análise de redes sociais: cartografando topologias e temporalidades em rede. Anais da Compós. Goiânia/GO, 2016.

. Entrevista com Fábio Malini. Parágrafo, v. 5, n. 2, 2017.

MATHIEU, D. The Continued Relevance of Reception Analysis in the Age of Social Media. Tripodos, v. 36, 2015.

MOROZOV, E. Big Tech: a ascensão dos dados e a morte da política. São Paulo: Ubu, 2018.

MOSCO, V. To the Cloud: Big Data in a Turbulent World. Nova lorque: Paradigm, 2014.

NOBLE, S. Algorithms of Opression: how search engines reinforce racism. Nova lorque: NYU Press, 2018.

PIENIZ, M.; SILVA, R.; MATOS, L. Sujeitos em trânsito na internet. In: JACKS, N. et ali. Meios e Audiências III. Porto Alegre: Sulina, 2017, p. 19-40.

PHILIPS, W.; MILNER, R. The Ambivalent Internet. Londres: Polity, 2017.

RECUERO, R.; BASTOS, M.; ZAGO, G.. Análise de Redes para Mídia Social. Porto Alegre: Sulina, 2015.

RICO, A. Fans of Columbine Shooters Eric Harris and Dylan Klebold. Transformative Workers and Cultures, v. 20, 2015

SAVAGE, M. The 'Social Life of Methods': a critical introduction. Theory, Culture \& Society, v. 30, n. 4 , p. 3-21, 2013.

SBARDELOTTO, M. Circulação em rede: a comutabilidade dos polos de produção e recepção no fluxo comunicacional digital. Anais da Compós. São Paulo: Cásper Líbero, 2017. Disponível em: <http://www.compos.org.br/data/arquivos_2017/trabalhos_ arquivo_3PEPPTB39151EKLTF3LY_26_5769_21_02_2017_08_39_18.pdf>. Acesso em: 20 fev. 2018. 
SCHRADIE, J. Big Data is Too Small: research implications of class inequality for online data collection. In: DEERY, J.; PRESS, A. (org.). Media and Class: TV, Film and Digital Culture. Abingdon: Taylor \& Francis, 2017, p. 200-213.

SHIFMAN, L. Memes in Digital Culture. Cambridge: MIT Press, 2014.

SILVERSTONE, R. Por que estudar a mídia? São Paulo: Loyola, 2002.

. Media and Morality. Cambridge: Polity Press, 2006.

SRNICEK, N. Platform Capitalism. Londres: Polity, 2017.

VAN DIJCK, J. Datafication, dataism and dataveillance: Big Data between scientific paradigm and ideology. Surveillance \& Society, v. 12, n. 2, 2014.

VOLOCHINOV, V. Marxismo e Filosofia da Linguagem. São Paulo: Ed. 34, 2017.

ZAGO, G. Circulação jornalística potencializada: o Twitter como espaço para filtro e comentário de notícias por interagentes. Comunicação \& Sociedade, v. 34, n. 1, 2012.

Artigo recebido em 19/11/2018

e aprovado em 04/03/2019. 